\title{
Konsonanz oder Dissonanz - die Diskussionslage der Umweltrisikobewertung von Industriechemikalien, gesehen aus der Sicht eines Vertreters der chemischen Industrie
}

\author{
Norbert Caspers
}

Erhalten: 19. Februar 2009/Akzeptiert: 30. April 2009/Online veröffentlicht: 20. Mai 2009

(C) Springer-Verlag 2009

\begin{abstract}
Zusammenfassung Ausgangspunkt des folgenden, naturgemäß subjektiven Meinungsbildes sind persönliche Erfahrungen, die der Autor im Laufe der vergangenen Jahre auf dem Gebiet der Entwicklung ökotoxikologischer Testmethoden und der regulatorischen Bewertungen hochvolumiger Industriechemikalien gemacht hat.
\end{abstract}

Schlüsselwörter Industriechemikalien · ökotoxikologische Testmethoden $\cdot$ Risikobewertung (Umwelt)

\begin{abstract}
This paper reflects various personal experiences of the author over the last years, concerning ecotoxicological test development and regulatory-type evaluations of the environmental risk assessment of high production volume chemicals.
\end{abstract}

Keywords Industrial chemicals - ecotoxicological test methods $\cdot$ environmental risk assessment

\section{Die Situation in Deutschland}

Auf den ersten Blick erscheint es, dass es in diesem Kontext nicht viel Kontroverses zu beklagen gibt. Meiner Wahrnehmung nach gibt es in Deutschland im Dialog zwischen Behörden und den Firmen bzw. dem Verband der Chemischen Industrie (VCI) eine funktionierende Kommunikationsschiene und eine faire Streitkultur (z. B. SETAC GLB 2005; Länge

Verantwortliche Herausgeber: Tobias Frische $\cdot$ Jan Ahlers · Bettina Hitzfeld

N. Caspers $(\bowtie)$

Currenta GmbH \& Co. OHG, Analytik/Produktsicherheit,

Gebäude Q18, 51368 Leverkusen, Deutschland

E-Mail: Norbert.Caspers@currenta.de et al. 2006; Gies 2006). Dazu trägt u. a. die regelmäßige Ausrichtung von Workshops und Fachgesprächen mit fachlichen Schwerpunktthemen bei, die im Vorfeld gesetzlicher Regelungen alle beteiligten Stakeholder zusammenrufen, um - soweit wie möglich - einen inhaltlichen Konsens zu erreichen.

Ein zweites Element der guten Zusammenarbeit zwischen Behörden und Industrie sehe ich auf dem Gebiet der ökotoxikologischen Methodenentwicklung, wie sie auf OECDund ISO-Ebene etabliert ist. Im Rahmen des „OECD - Test Guidelines Programme“ sucht (und findet) die nationale Koordinationsstelle Deutschlands, angesiedelt im Umweltbundesamt, das Votum der nationalen Experten, bevor Folgeaktivitäten auf der OECD-Ebene angestoßen werden.

In die Standardisierungsvorhaben auf ISO-Ebene - etwa im Rahmen des Technical Committee 147 (Water Quality) sind neben Vertretern der nationalen Normungsinstitute und Behördenvertretern ebenfalls Experten der chemischen Industrie mit ihrem Sachverstand eingebunden. Auch hier hat sich das Prinzip bewährt, zunächst im internen Zirkel die nationale Position zu entwickeln - und dabei keine Meinungsunterschiede auszuklammern -, um das Resultat dann auf internationaler Ebene „mit einer Zunge“ gemeinsam vorzutragen.

Dies alles funktioniert in weitgehend reibungsfreier Form nur, wenn „,man miteinander kann“; Voraussetzung hierfür es soll durchaus noch ein zweites Mal betont werden - sind wechselseitige Fairness und Offenheit, gerade in strittigen Fragen, als wichtige vertrauensbildende Maßnahmen.

Alles paletti also? Ja und nein! Ja, weil ich glaube, dass die Prozesse der Meinungsbildung, Problembehandlung und Konsensfindung - wie vorstehend geschildert - im nationalen Rahmen zufriedenstellend ablaufen. Nein, weil es auf europäischer Ebene bei eben diesen Prozessen, und insbesondere bei der ökotoxikologischen Stoffbewertung, erhebliche Defizite gibt. Dies wiegt besonders schwer, da die Risikobewer- 
tung von Industriechemikalien im Rahmen der EU-AltstoffVerordnung (die kürzlich dem neuen EU-Chemikalienrecht REACH Platz gemacht hat) eben nicht auf nationalstaatlicher, sondern auf europäischer Ebene vorgenommen wurde.

\section{Die Situation auf europäischer Ebene}

Genau hier setzt das entscheidende Problem ein: einigen nationalen Repräsentanten der EU-Mitgliedsländer fehlt die fachliche und (englisch-) sprachliche internationale Erfahrung. Dies führte in den „Technical Meetings“, in denen die Risikobewertungen von HPV-Chemikalien (HPV = high production volume) behandelt wurden, gelegentlich dazu, dass eine „vokale“ Minderheit einiger weniger EU-Länder einer schweigenden Mehrheit der übrigen Länder gegenüberstand, die sich schwer taten, eine eigene fundierte Meinung zu artikulieren. Die resultierenden Ungleichgewichtungen bei der Bewertung hochvolumiger Industriechemikalien sind aus meiner Sicht mehr als bedenklich, werden hier doch richtungsgebende Entscheidungen über das Wohl und Wehe ökonomisch wichtiger Chemikalien und die Erfordernisse des Umwelt- und Gesundheitsschutzes getroffen. Ich hege die Hoffnung, dass diese Situation nicht nur für die industriellen Produzenten, sondern auch für die Vertreter engagierter EU-Mitgliedsländer inakzeptabel ist, denn sie unterminiert die fachliche Reputation des gesamten „Technical Committee“. In diesem Sinne wäre es sehr wünschenswert, wenn das deutsche Umweltbundesamt seinen Einfluss geltend machen würde, auf solche Missstände verstärkt aufmerksam zu machen, um sie künftig abzustellen.

Die Risikobewertungen im Rahmen der EU-AltstoffVerordnung sind zwar mit Inkrafttreten von REACH passé; aber auch das neue Europäische Chemikalienrecht wird, zumindest im Rahmen des Zulassungsverfahrens, Strukturen und Prozesse für eine ausgewogene und die Mitgliedsländer integrierende Stoffbewertung benötigen.

Neben diesen eher prozessuralen Schwachstellen gab es bei den Technical Meetings zur Risikobewertung von Industriechemikalien einige „Evergreens“ an fachlich-inhaltlichen Defiziten, die vermutlich weit in die REACH-Zeit hineinwirken werden. So ist es äußerst bedauernswert, dass bis in die jüngste Vergangenheit Instrumentarien wie „Read across" - von REACH ausdrücklich als geeignetes Mittel zur Bewältigung der Sisyphus-Aufgabe Stoffbewertung empfohlen (vgl. Ahlers et al. 2008) - nicht, bzw. allenfalls sehr zögerlich im Rahmen des Environmental Risk Assessment eingesetzt wurden. Das gleiche galt lange Zeit für den Einsatz der „Species Sensitivity Distribution“ (SSD) chronischer NOECs zur PNEC-Ableitung bei datenreichen organischen Industriechemikalien; erst kürzlich wurde dieser Ansatz zum ersten Mal, und zwar durchaus erfolgreich, zur Anwendung gebracht.
Eine besonders irritierende Diskussion entspann sich häufig im Kontext von Testanforderungen und Studiendesign bei chemischen Stoffen mit unterstelltem östrogenähnlichen Potenzial: Angesichts der inhärenten biologischen Variabilität bestimmter Endpunkte (z. B. Vitellogenin; Secondary Sex Characteristics) mag der Wunsch nach Verdopplung oder Verdreifachung oder Verzehnfachung (etc.) der Replikatenzahlen in chronischen Tests theoretisch nachvollziehbar sein; der im ähnlichen Kontext - durchaus von den gleichen Personen - gerne geäußerte Wunsch nach größtmöglicher Reduzierung der Tierzahlen wirkte denn doch etwas befremdlich. Ein tieferes Verständnis dafür, dass bei komplexen und langwierigen Studien mit einer Vielzahl konventioneller und endokriner Endpunkte selbst international renommierte Prüflaboratorien an natürliche Kapazitätsgrenzen stoßen, war nach meinen Erfahrungen einigen technischen Experten der EU-Mitgliedsländer kaum zu vermitteln.

Ich betone gerne noch einmal, dass die vorstehenden Textpassagen deshalb im Präteritum geschrieben wurden, weil es sich um historische Erfahrungen mit der EU-Altstoff-Verordnung handelt, von denen ich hoffe, dass sie in der (REACH-)Zukunft nicht mehr im Präsens beschrieben werden müssen.

\section{Wissenschaftliche Forschung - Qualität und Glaubwürdigkeit}

Soweit meine Anmerkungen zum Dialog zwischen Behörden und chemischer Industrie. Die Auseinandersetzung mit der Risikobewertung von Industriechemikalien wird jedoch tatsächlich im Trialog geführt, an dem als dritter Interessenvertreter die wissenschaftliche Gemeinschaft teilnimmt. Die Beobachtung der nationalen und internationalen Forschungslandschaft, insbesondere im universitären Bereich, vermittelt derzeit den Eindruck, dass die Ökotoxikologie - um es vorsichtig auszudrücken - nicht gerade an vorderster Position der Hitparade populärer (und damit als förderungswürdig erachteter) biologischer Teildisziplinen rangiert. Etwas drastischer, aber vielleicht doch treffend (und hoffentlich selbsterklärend) wurde die Situation der forschenden und lehrenden Ökotoxikologie in Deutschland anlässlich eines UBA-Workshops im Jahre 2002 von einem Hochschulvertreter als „embryotoxisch" bezeichnet. Möglicherweise sind Begriffe wie „Normung“, „standardisierte Testverfahren“, „Versuchsplanung“, ,historische Datenbasis“" und „Wiederholungsgenauigkeit" nicht leicht mit dem derzeitigen Erwartungshorizont für hochwertige Forschung in Einklang zu bringen - zumal in Zeiten knapper Kassen, in denen der Rubel der Forschungsförderung in Form von Drittmitteln langsamer rollt. In diesem für die wachsende Zahl der forschenden Ökotoxikologen zunehmend schwierigeren Umfeld steigt der Selektionsdruck, sich durch rasche Präsentation und Publikation 
neuartiger Befunde mit „populären“ Chemikalien zu qualifizieren. Zumal Drittmittelgeber - m.E. zu Recht - nach Jahren intensiver Forschungsförderung von Ergebnisberichten etwas mehr erwarten als lapidare Schlussformeln wie „weiterer Forschungsbedarf ist vonnöten, um offene Punkte abzuklären“. Ob bei einer derartigen Ausgangssituation die Qualitätsanforderungen an regulatorisch verwendbare chronische Studien (z.B. zielgerichtete Versuchsplanung; chemisch-analytische Expositionsüberwachung; Qualitätskontrolle) strikt eingehalten werden, wäre nach meinem Dafürhalten sorgfältiger zu prüfen, als dies zurzeit geschieht. Diese Anforderung muss insbesondere gelten, wenn Befunde mit neuen Endpunkten bzw. neuen Testspezies außerhalb des etablierten OECD-Repertoires generiert werden.

In der gelegentlich etwas verhärteten Diskussion über Qualitätsanforderungen an regulatorisch bedeutsame ökotoxikologische Studien wird oft ein Fragezeichen hinter den Wert und die Glaubwürdigkeit industrieller bzw. seitens der Industrie kontraktierter Studien gestellt - bis hin zu der Unterstellung, dass „Gefälligkeitsergebnisse“ kreiert werden, die ausschließlich die Argumentationslage der Industrie unterstützen sollen. In Anbetracht des dichten Netzes interner und externer Audits, die in puncto Qualitätssicherung und Guter Laborpraxis (GLP), insbesondere bei chronischen Laborstudien, durchgeführt werden, sowohl in Industrielaboratorien als auch in den Labors kommerzieller Auftragsforschungsinstitute, verweigere ich mich - um den Pfad der Höflichkeit nicht zu verlassen - einer vertieften Diskussion dieses Punktes. Aber ich hoffe, meine Position ist trotzdem hinreichend sichtbar geworden.

Im Übrigen verweise ich gerne auf den ausgezeichneten Übersichtsvortrag zu dieser Thematik, der auf der 16. Jahrestagung von SETAC Europe in Den Haag gegeben wurde (Purchase 2006). Vielleicht etwas zusammenhanglos - aber eigentlich passt dieser Hinweis immer - das Bekenntnis zum „Weight-of-Evidence“-Approach, der mittlerweile in viele Bereiche der Umweltdiskussion, so auch in das Gesetzeswerk REACH als leitendes Prinzip, Eingang gefunden hat. Alle diejenigen, die valide Daten für eine regulatorische Bewertung von Chemikalien zur Verfügung stellen - ob aus der wissenschaftlichen Forschung, der Industrie oder aus Behördenlaboratorien - müssen akzeptieren, dass sie keinen Alleinvertretungsanspruch auf die ,wissenschaftliche Wahrheit" (die möglicherweise Thema eines separaten Diskussionsbeitrages sein könnte) haben, sondern dass sich die regulatorische Bewertung erst aus der Zusammenschau aller verfügbaren Informationen ableitet.

\section{Eine Empfehlung und ein Ausblick}

Zum Abschluss meines Diskussionsbeitrages würde ich gerne anregen, dass sich der Trialog von Regulatoren, wis- senschaftlicher Forschung und Industrie eines Themas annehme, das es aus meiner Sicht wert ist, näher betrachtet zu werden und das die Entwicklung der regulatorischen Ökotoxikologie vorantreiben könnte.

Eine der Kernaufgaben der regulatorischen Ökotoxikologie, wie im Vorwort dieser Beitragsserie von Frische et al. (2009) treffend formuliert, ist „... Bewertungsstrategien zu etablieren, welche auf der Basis umfangreicher und oft komplexer Informationen fundierte und nachvollziehbare Entscheidungen zu Gefahren und Risiken von Chemikalien ermöglichen." Um diesem hohen Anspruch zu genügen, müssen wir, wie von Sumpter und Johnson (2005) in einem lesenswerten Artikel deutlich gemacht, uns zunächst mit der scheinbar einfachen Frage „Was ist normal?" auseinandersetzen, bevor wir uns im zweiten Schritt der Frage „Was ist unnormal?“ (und „Warum ist es unnormal?“) zuwenden. Ich wage zu behaupten, dass wir bei einer Vielzahl „neuer Endpunkte“ noch keine klare Vorstellung haben, wie der Bereich der Normalität - dessen Abbildung wir ja dann in der Negativkontrolle eines Tests erwarten dürfen - zu quantifizieren, vielleicht nicht einmal wie er zu qualifizieren ist. Ein Beispiel zur Verdeutlichung: Der sorgfältige Review-Artikel von Grim et al. (2007) hat am Beispiel des Medaka (Oryzias latipes) deutlich gemacht, dass eine gewisse Inzidenz an Intersex (,testicular oocytes" und „ovarian testicular tissue“) unter unkontaminierten Bedingungen auftritt und damit so etwas wie Normalität in einer Medaka-Population widerspiegelt, ohne dass man gleich östrogene Effekte durch Xenobiotica bemühen müsste (bzw. darf). Ich wage zu behaupten, dass bei sorgfältiger Untersuchung ähnliche Überraschungen bei einer Reihe weiterer Testspezies und weiterer Endpunkte anstehen. Vielleicht wäre es die Sache wert, einige graue Zellen und einige Euros in die Beleuchtung dieser Fragestellung zu investieren; sie könnte helfen, eine Präzisierung der Null-Linie für adverse Effekte vorzunehmen. Den Startpunkt könnte eine Diskussion auf einer Jahrestagung von SETAC-GLB markieren, seit jeher ein geeignetes Forum zur Diskussion innovativer Ansätze.

Eine Botschaft, die ich gerne noch vermitteln möchte; vielleicht eignet sie sich sogar anstelle eines zusammenfassenden Schlusswortes.

Wir sind - umwelttoxikologisch gesprochen - weit entfernt vom Untergang des Abendlandes, wenn uns das auch einige notorische Angstmacher weiterhin glauben lassen wollen. Ganz im Gegenteil; es mehren sich die Zeichen, dass die vereinten Anstrengungen der Gesprächspartner in der Umweltdiskussion schon zu erheblich verbesserten Umweltsituationen geführt haben (und weiterhin führen werden), und zwar in allen Kompartimenten. So zeigen es jedenfalls die ökologisch anspruchsvollen Tier- und Pflanzenarten, die nach Zeiten der Zurückhaltung mehr und mehr $\mathrm{Fu}$ gefasst haben in unseren Ökosystemen und damit deut- 
lich signalisieren, wie es um die Qualität ihrer Lebensräume bestellt ist. Und das ist es letzten Endes doch, was die regulatorische Ökotoxikologie erreichen will.

\section{Literatur}

SETAC GLB (2005) „Prozesse verstehen - Risiken bewerten“. Runder Tisch „Regulatorische Ökotoxikologie zwischen Wissenschaft und PNEC“, 10. Tagung SETAC GLB, 28.-30. September 2005, Basel

Ahlers J, Stock F, Werschkun B (2008) Integrated testing and intelligent assessment - new challenges under REACH. Environ Sci Pollut Res 15:565-572

Frische T, Ahlers J, Hitzfeld B (2009) Kochsalz verbieten? Ein nachdenkliches Vorwort zur Beitragsserie „Regulatorische Ökotoxikologie“. Umweltwiss Schadst Forsch 21:1-4
Gies A (2006) Anmerkungen zum Beitrag von Reinhard Länge et al. „Kriterien zur Bewertung der Qualität und Validität von toxikologischen und ökotoxikologischen Studien für regulatorische Fragestellungen." Umweltwiss Schadst Forsch 18:55-56

Grim KC, Wolfe M, Hawkins W, Johnson R, Wolf J (2007) Intersex in Japanese Medaka (Oryzias latipes) used as negative controls in toxicologic bioassays: a review of 54 cases from 41 studies. Environ Toxicol Chem 26:1636-1643

Länge R, Caspers N, Ensenbach U, Pallapies D, Zok S (2006) Kriterien zur Bewertung der Qualität und Validität von toxikologischen und ökotoxikologischen Studien für regulatorische Fragestellungen. Umweltwiss Schadst Forsch 18:49-54

Purchase IFH (2006) Ethics concerning experimental (eco)toxicological research. Keynote 16th Annual Meeting SETAC Europe in The Hague, Netherlands, 7-11 May 2006

Sumpter JP, Johnson AC (2005) Lessons from endocrine disruption and their application to other issues concerning trace organics in the aquatic environment. Environ Sci Technol 39:4321-4332 